\title{
Extreme ultraviolet detection using AIGaN-on-Si inverted Schottky photodiodes
}

\author{
Pawel E. Malinowski, ${ }^{1,2, a)}$ Jean-Yves Duboz, ${ }^{3}$ Piet De Moor,${ }^{1}$ Kyriaki Minoglou, ${ }^{1}$ \\ Joachim John, ${ }^{1}$ Sara Martin Horcajo, ${ }^{1}$ Fabrice Semond, ${ }^{3}$ Eric Frayssinet, ${ }^{3}$ \\ Peter Verhoeve, ${ }^{4}$ Marco Esposito, ${ }^{4,5}$ Boris Giordanengo, ${ }^{6}$ Ali BenMoussa, ${ }^{6}$ \\ Robert Mertens, ${ }^{1,2}$ and Chris Van Hoof ${ }^{1,2}$ \\ ${ }^{1}$ IMEC, Kapeldreef 75, B-3001 Leuven, Belgium \\ ${ }^{2}$ ESAT, K.U. Leuven, B-3001 Leuven, Belgium \\ ${ }^{3}$ CNRS/CRHEA, rue Bernard Gregory, F-06560 Valbonne, France \\ ${ }^{4}$ ESA/ESTEC, Keplerlaan 1, 2200 AG Noordwijk, The Netherlands \\ ${ }^{5}$ Cosine, Niels Bohrweg 11, 2333 CA Leiden, The Netherlands \\ ${ }^{6}$ Royal Observatory of Belgium, Ringlaan 3, B-1180 Brussels, Belgium
}

\begin{abstract}
We report on the fabrication of aluminum gallium nitride ( $\mathrm{AlGaN}$ ) Schottky diodes for extreme ultraviolet (EUV) detection. AlGaN layers were grown on silicon wafers by molecular beam epitaxy with the conventional and inverted Schottky structure, where the undoped, active layer was grown before or after the n-doped layer, respectively. Different current mechanisms were observed in the two structures. The inverted Schottky diode was designed for the optimized backside sensitivity in the hybrid imagers. A cut-off wavelength of $280 \mathrm{~nm}$ was observed with three orders of magnitude intrinsic rejection ratio of the visible radiation. Furthermore, the inverted structure was characterized using a EUV source based on helium discharge and an open electrode design was used to improve the sensitivity. The characteristic He I and He II emission lines were observed at the wavelengths of $58.4 \mathrm{~nm}$ and $30.4 \mathrm{~nm}$, respectively, proving the feasibility of using the inverted layer stack for EUV detection.
\end{abstract}

Extreme ultraviolet (EUV) detection is gaining increasing attention with recent developments in solar science and EUV lithography. ${ }^{1,2}$ Photodetectors used in most applications are based on $\mathrm{Si}$, sensitive to the visible and infrared radiation. Using wide bandgap semiconductors as the active layer can reduce the number of filters required to suppress the unwanted radiation at large wavelengths. ${ }^{3-7}$ Furthermore, an improved EUV hardness is observed in devices based on this compound. ${ }^{8}$ Recent advances in epitaxial growth enable fabrication of high quality aluminum gallium nitride $(\mathrm{AlGaN})$ layers on $\mathrm{Si}$ substrates, both by molecular beam epitaxy (MBE) and metal-organic chemical vapor deposition. For advanced applications, two-dimensional arrays with $\mathrm{AlGaN} \mathrm{ac-}$ tive layers are highly desired, which require special design both of the pixel and of the layer stack. ${ }^{9}$ In this paper we present the inverted Schottky structure in submicron-thin $\mathrm{Al}$ GaN layers designed especially for backside illuminated two-dimensional arrays with a very small pixel-to-pixel pitch. The conventional Schottky structure is not suitable because of detrimental absorption and recombination in the doped layer when the device is illuminated from the backside, as all materials are highly absorbing in the EUV. ${ }^{10}$

Active AlGaN layers were grown on 2 in. $\mathrm{Si}(111)$ wafers by MBE. Two layer types were investigated: a conventional Schottky with n-doped layer at the bottom of the stack ${ }^{11}$ and an inverted Schottky with the n-doping at the top (Fig. 1). The growth started with $40 \mathrm{~nm}$ of AlN nucleation layer. The growth continued for $300 \mathrm{~nm}$ while gradually decreasing the Al content from $100 \%$ to $40 \%$. Furthermore, the Si doping was introduced for either first (conventional) or last (in-

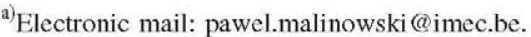

verted) $100 \mathrm{~nm}$ with fixed $\mathrm{Al}$ content. This yielded a nonintentionally doped $\mathrm{AlGaN}$ layer serving as the active layer of the photodetector $(300 \mathrm{~nm})$ with an n-doped layer to improve the Ohmic contact $(100 \mathrm{~nm})$. Processing started with accessing the bottom layer by $\mathrm{Cl}$-based reactive ion etching (RIE) of approximately $200 \mathrm{~nm}$. In the conventional Schottky, undoped $\mathrm{AlGaN}$ was left as islands (mesa), whereas in the inverted Schottky it was accessed in the etched pits (inverted mesa). The Ohmic contact was a sputtered metal stack of $\mathrm{Ti} / \mathrm{Al} / \mathrm{Mo} / \mathrm{Au}(10 / 40 / 25 / 50 \mathrm{~nm}$, respectively), which was subsequently annealed for $1 \mathrm{~min}$ at $850^{\circ} \mathrm{C}$. The contact resistance of approximately $1 \Omega \mathrm{mm}$ was extracted from the transfer length method measurements using large Ohmic contacts spaced from 32 to $1 \mu \mathrm{m}$ for both layers. The Schottky contact was a semitransparent, $20 \mathrm{~nm} \mathrm{Au} \mathrm{layer.}$ Additionally, $100 \mathrm{~nm}$ of $\mathrm{SiO}_{2}$ was used as passivation and to separate the Ohmic and fanout metallization. The passivation was opened by $\mathrm{SF}_{6}$-based RIE over the active area and the contact level was deposited (TiW/Ni/Au, 10/150/150 nm, re-
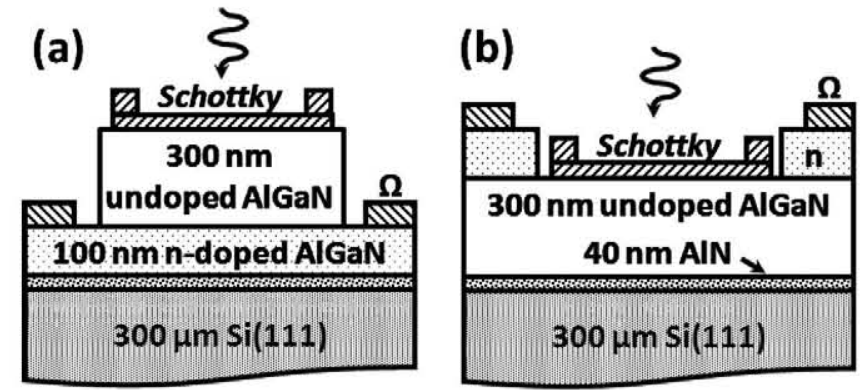

FIG. 1. AlGaN EUV photodetector structure: conventional (a) and inverted Schottky (b). 


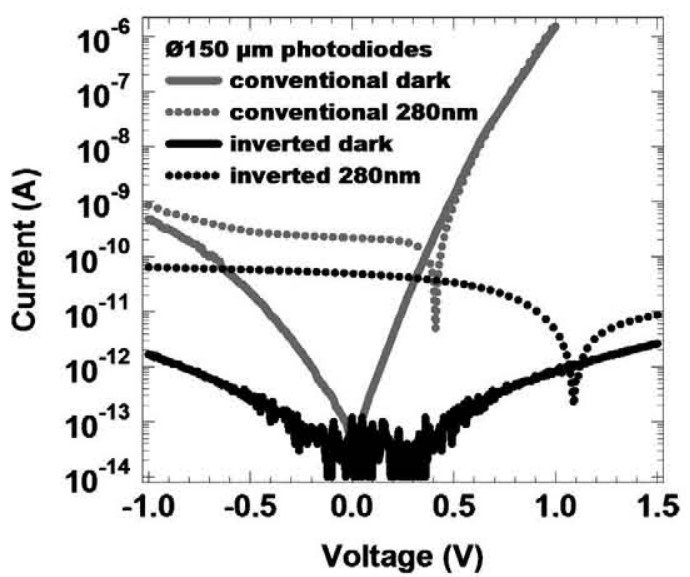

FIG. 2. Current-voltage characteristics of the conventional (lighter) and inverted (darker) Schottky photodiodes in darkness (solid line) and under illumination with the wavelength of $280 \mathrm{~nm}(5 \mu \mathrm{W}$, dotted line).

spectively). In the inverted structure, the Schottky contact had a ring shape $(5 \mu \mathrm{m}$ width) which allowed direct exposure of the active layer to the incoming radiation. A top view optical microscope image is shown in the inset of Fig. 4, with the round Ohmic surrounding the mesa region and the exposed active area in the middle. The fanout metallization crosses the Ohmic ring on top of the passivation.

Samples were characterized at room temperature using a Keithley 236 Source Measure Unit (SMU) with dedicated low-current probes. Current-voltage curves of the representative devices for the conventional and inverted Schottky diodes (Fig. 2) differed significantly, even though the Schottky contact, the contact resistance of the Ohmic contact and the active layers were the same. In darkness, it was observed that the magnitude of the forward current in the inverted structure does not exceed the magnitude of the reverse current, resembling the behavior of a metal-semiconductor-metal (MSM) device. The current levels were lower than $2 \mathrm{pA}$ for a $150 \mu \mathrm{m}$ circular diode in both polarities. In contrast, the conventional Schottky showed a much higher reverse current (almost $1 \mathrm{nA}$ at $-1 \mathrm{~V}$ ) together with a forward current at $1 \mathrm{~V}$ approximately four decades higher. To investigate the photosensitivity, the devices were illuminated with radiation produced by a $150 \mathrm{~W}$ Xe lamp with filters for wavelengths between 200 and $400 \mathrm{~nm}$. Figure 2 shows the photocurrent curves under illumination with the wavelength of $280 \mathrm{~nm}$ (power of several $\mu \mathrm{W} / \mathrm{cm}^{2}$ ), which was the expected cut-off wavelength of the active layer used $\left(\mathrm{Al}_{0.4} \mathrm{Ga}_{0.6} \mathrm{~N}\right)$. Interestingly, the photocurrent curve is characteristic of a photovoltaic detector, with a strong response at $0 \mathrm{~V}$ also for the inverted structure. If the device was in fact an MSM, the photocurrent would be zero with no bias. Instead, it behaves as a Schottky diode with a very poor forward current. This behavior can be explained by the carrier collection in both layer structures (Fig. 3). The conventional structure [Fig. 3(a) ] can be considered a quasivertical diode, where the electron injection from the Ohmic contact takes place from the entire area underneath the Schottky contact, since the doped layer is at the bottom. In the inverted structure [Fig. 3(b)], the electrons are injected only from the perimeter of the Schottky contact, since the doped layer is etched to access the active area and only surrounds it at a certain distance (micrometers). The same applies for the collection of the photogenerated carriers and is significant especially for the
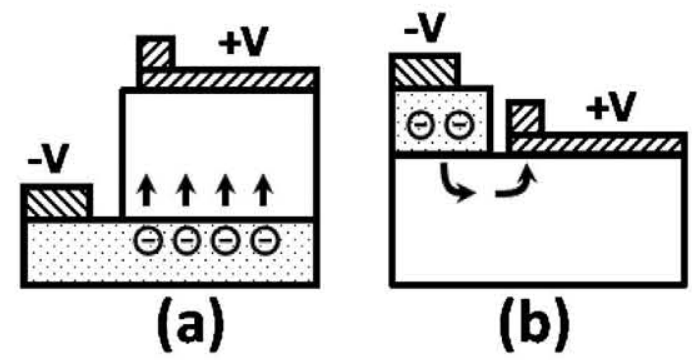

FIG. 3. Carrier collection under forward bias in the conventional (a) and inverted Schottky diode (b).

large devices. The lateral geometry of the inverted structure introduces an access resistance which limits the current. However, in the view of the designed small-pitch arrays (the final goal is 10 to $30 \mu \mathrm{m}$ pixel-to-pixel pitch) this issue should not detrimentally influence the carrier collection efficiency.

The photocurrent values taken at $0 \mathrm{~V}$ were used to calculate the spectral responsivity (Fig. 4). The average responsivity of approximately $20 \mathrm{~mA} / \mathrm{W}$ was recorded at the cut-off wavelength $(280 \mathrm{~nm})$ for sets of more than ten devices with different diameters (40 up to $300 \mu \mathrm{m}$ ) fabricated with two layer structures. This relatively low responsivity $(5 \mathrm{~mA} / \mathrm{W}$ for the $150 \mu \mathrm{m}$ diodes) was attributed to the active layer design, optimized for the EUV range. The thickness of only $300 \mathrm{~nm}$ was not optimum for detection of the higher wavelengths and also resulted in higher access resistance of the diodes. Nevertheless, the rejection ratio of the visible radiation $(400 \mathrm{~nm})$ was at least three orders of magnitude since the photocurrent at $400 \mathrm{~nm}$ was below the measurement limit. The response at $300 \mathrm{~nm}$ comes from a relatively high bandwidth of the filters used $(10 \mathrm{~nm}$ full width at half maximum). Additionally, Fig. 4 shows a photocurrent curve at 0 $\mathrm{V}$ measured by a $150 \mu \mathrm{m}$ device using the synchrotron radiation at Physikalisch-Technische Bundesanstalt (PTB, Berlin), in the wavelength range of 200 to $310 \mathrm{~nm}$. It confirms the shape of the response and shows a much sharper cut-off at $280 \mathrm{~nm}$.

EUV sensitivity was tested using a beamline based on a glow discharge chamber pumped with $\mathrm{He}^{12}$ After aligning the detector in the center of the beam, the EUV McPherson monochromator was used to scan through the wavelengths from 13 to $65 \mathrm{~nm}$. Figure 5 shows that it was possible to

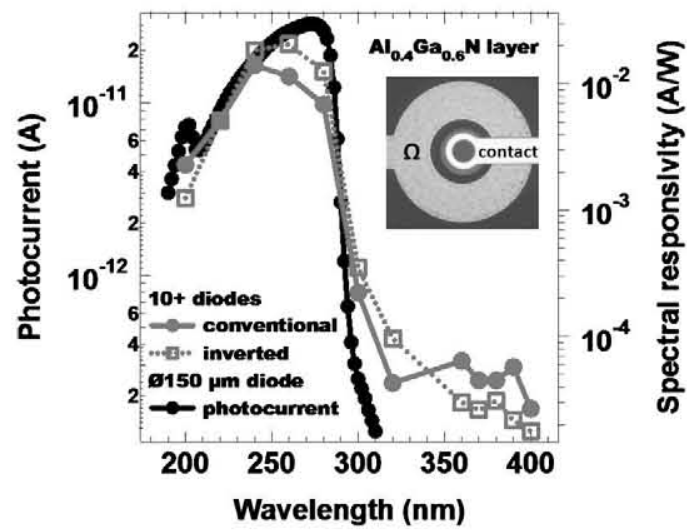

FIG. 4. (Color online) Average spectral responsivity of the conventional and inverted Schottky photodiodes measured with the Xe lamp (gray) compared to the photocurrent measured using the synchrotron radiation (black). The inset shows the open electrode design. 


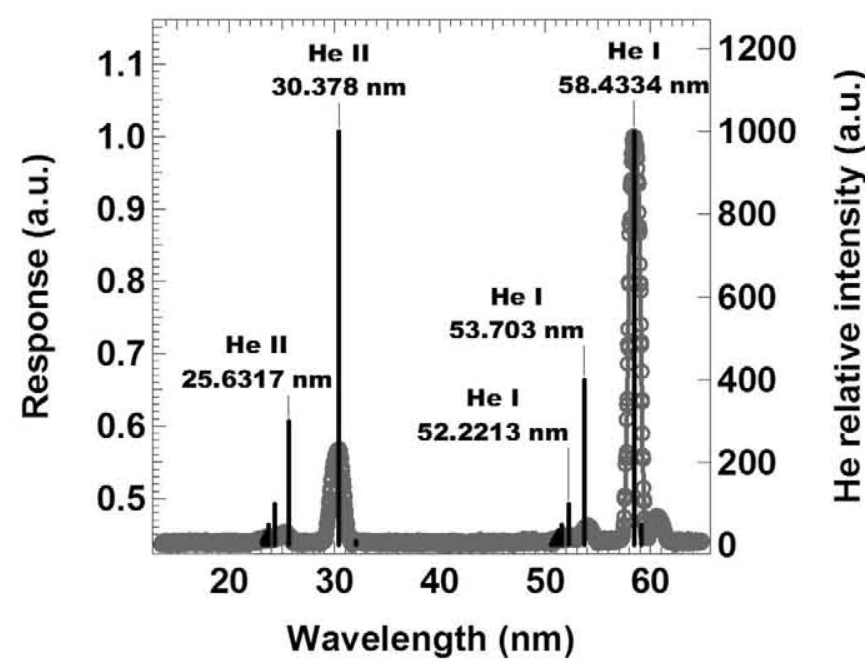

FIG. 5. AlGaN Schottky photodiode response (gray line) compared with the He discharge relative intensity (black sticks, after Ref. 13).

observe two main emission lines: He II (at $30.4 \mathrm{~nm}$ ) and $\mathrm{He}$ I (at $58.4 \mathrm{~nm}$ ). The measurement was performed using fully open slits (to maximize the irradiance) and repeated with reduced slits. In the latter configuration, it was possible to observe more peaks and even the second order peak of the He II emission at $60.8 \mathrm{~nm}$. Another He II peak could be noticed around $25 \mathrm{~nm}$ as well as two smaller peaks corresponding to $\mathrm{He} \mathrm{I}$ at 52.2 and $53.7 \mathrm{~nm}{ }^{13}$ It should be noted that in the vicinity of the He I emission, there is a significant contribution of photoemission from both $\mathrm{Au}$ contacts and the GaN surface. The higher response at $58.4 \mathrm{~nm}$ compared to $30.4 \mathrm{~nm}$ could be partly attributed to the additional signal due to the surface photoelectric effect. ${ }^{14}$

Since the power of the discharge source was very low $(<400 \mathrm{pW}$ measured with a $5 \mathrm{~mm}$ diameter Si-diode), it was crucial to choose an appropriate photodiode design. In the experiment described, the Schottky contact was partially open above the active layer of the device, which allows penetration of the incoming radiation directly into the AlGaN layer. Moreover, special care was taken to keep the surface clean during processing. Devices with a Schottky contact consisting of a continuous layer of metal were also fabricated for comparison, since it was observed in previous works that an electrode with minimum perimeter is beneficial for the leakage current decrease ${ }^{15}$ However, even a very thin, semitransparent Au layer (10-15 nm) deposited in the same $\mathrm{Al}$ GaN detector reduced the photocurrent below the detection limit. An improvement to this design would be using the backside illumination geometry, where the fill factor can achieve $100 \%$ with no shadowing due to the contact metallization. ${ }^{12,15} \mathrm{~A}$ key factor for processing of the backside illuminated devices is the fact that the $\mathrm{AlGaN}$ heterostructure is grown on an easily-removable $\mathrm{Si}$ substrate. Test vehicles to verify the backside sensitivity in EUV are in preparation.

In conclusion, we presented Extreme Ultraviolet photodetectors based on Schottky photodiodes, where the active layer is $\mathrm{AlGaN}$ with $40 \% \mathrm{Al}$ concentration. Such design provides a cut-off wavelength of $280 \mathrm{~nm}$ with a high intrinsic rejection ratio of the visible radiation. The conventional and inverted Schottky structures were investigated. Sensitivity in the EUV range was verified using a $\mathrm{He}$ glow discharge beamline. It was possible to detect the He I and He II emission lines. EUV sensitivity was improved by using a Schottky electrode with a ring design, where the active layer is directly exposed to the incoming radiation.

This work was supported by the European Space Agency under ESA/ESTEC Contract No. 19947/06/NL/PM, BOLD. The Authors would like to express their gratitude to Udo Kroth and Alexander Gottwald from PTB for the synchrotron measurements.

${ }^{1}$ P. Lemaire, K. Wilhelm, U. Schuhle, W. Curdt, A. I. Poland, S. D. Jordan, R. J. Thomas, D. M. Hassler, and J.-C. Vial, Adv. Space Res. 20, 2249 (1997).

${ }^{2}$ M. Razeghi, Proc. IEEE 90, 1006 (2002).

${ }^{3}$ E. Monroy, T. Palacios, O. Hainaut, F. Omnes, F. Calle, and J.-F. Hochedez, Appl. Phys. Lett. 80, 3198 (2002).

${ }^{4}$ J. Li, Z. Y. Fan, R. Dahal, M. L. Nakarmi, J. Y. Lin, and H. X. Jiang, Appl. Phys. Lett. 89, 213510 (2006).

${ }^{5}$ R. McClintock K. Mayes, A. Yasan, D. Shiell, P. Kung, and M. Razeghi, Appl. Phys. Lett. 86, 011117 (2005).

${ }^{6}$ T. Saito, T. Hitora, H. Ishihara, M. Matsuoka, H. Hitora, H. Kawai, I. Saito, and E. Yamaguchi, Metrologia 46, S272 (2009).

${ }^{7}$ A. BenMoussa, A. Soltani, U. Schühle, K. Haenen, Y. M. Chong, W. J. Zhang, R. Dahal, J. Y. Lin, H. X. Jiang, and H. A. Barkad, Diamond Relat. Mater. 18, 860 (2009).

${ }^{8}$ F. Barkusky, C. Peth, A. Bayer, K. Mann, J. John, and P. E. Malinowski, Rev. Sci. Instrum. 80, 093102 (2009).

${ }^{9}$ J.-L. Reverchon, S. Bansropun, J. A. Robo, J. P. Truffer, E. Costard, E. Frayssinet, J. Brault, F. Semond, J.-Y. Duboz, and M. Idir, Proc. SPIE 7474, 74741G (2009).

${ }^{10}$ P. E. Malinowski, J. John, J. Y. Duboz, G. Hellings, A. Lorenz, J. G. Rodriguez Madrid, C. Sturdevant, K. Cheng, M. Leys, J. Derluyn, J. Das, M. Germain, K. Minoglou, P. De Moor, E. Frayssinet, F. Semond, J.-F. Hochedez, B. Giordanengo, and R. Mertens, IEEE Electron Device Lett. 30, 1308 (2009).

${ }^{11}$ O. Katz, V. Garber, B. Meyler, G. Bahir, and J. Salzman, Phys. Status Solidi A 188,345 (2001).

${ }^{12}$ P. E. Malinowski, J. Y. Duboz, J. John, C. Sturdevant, J. Das, J. Derluyn, M. Germain, P. De Moor, K. Minoglou, F. Semond, E. Frayssinet, J. F. Hochedez, B. Giordanengo, C. Van Hoof, and R. Mertens, Proc. SPIE 7726, 772617 (2010).

${ }^{13}$ National Institute of Standards and Technology Database: http:// www.nist.gov/physlab/data/asd.cfm

${ }^{14}$ A. Motogaito, M. Yamaguchi, K. Hiramatsu, M. Kotoh, Y. Ohuchi, K. Tadatomo, Y. Hamamura, and K. Fukui, Jpn. J. Appl. Phys., Part 2 40, L.368 (2001).

${ }^{15}$ G. Hellings, J. John, A. Lorenz, P. Malinowski, and R. Mertens, IEEE Trans. Electron Devices 56, 2833 (2009). 